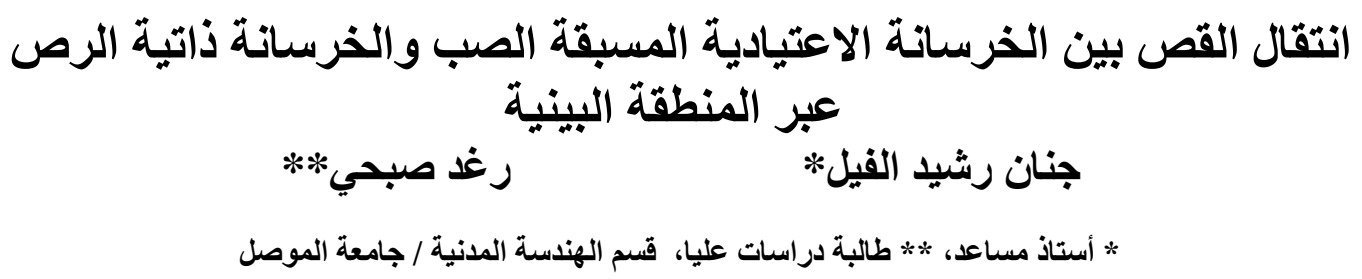

\title{
الخلاصة
}

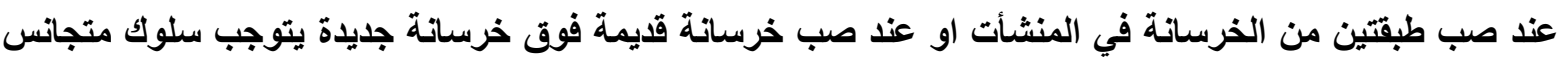

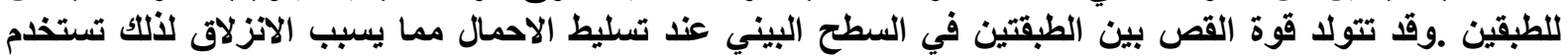

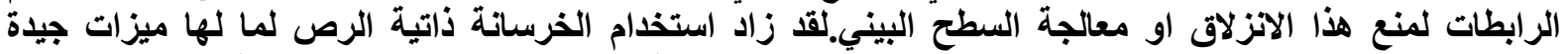

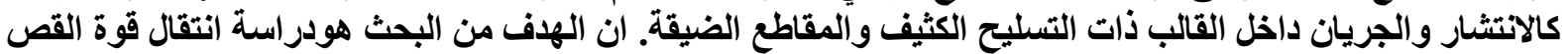

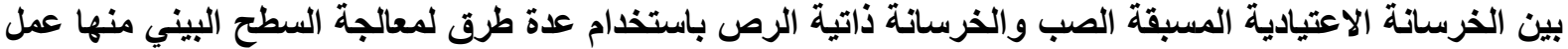

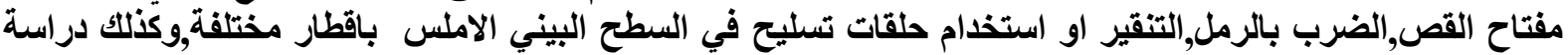

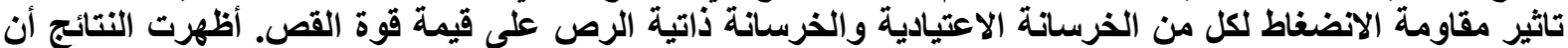

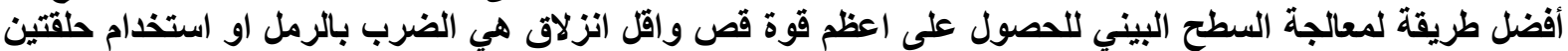

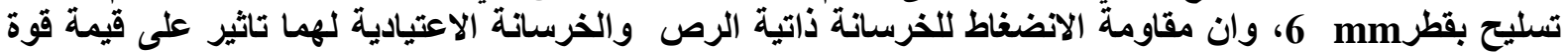

\section{Shear Transfer between Precast Normal Concrete and Self Compacting Concrete through the Interface Surface}

\author{
Janan Rasheed Al-Feel
}

Ragad Subhi

\begin{abstract}
When cast two different types of concrete in the structures or cast new concrete over old concrete it should be act monolithically. As the load applied a shear force between them will cause slip, so treatment or connectors should be used on the interface. The self compacting concrete (SCC) used widely due to its good properties as spreading and passing ability through congested reinforcement. The aim of the present research is to study the shear transfer between precast normal concrete and self compacting concrete by using several methods for surface treatment such as shear-key, sand-blast, chipping or using steel stirrups. And also to study the effect of the compressive strength on the shear force is carried out. The results were shown that the best treatment method of the interface surface to get high shear force and less slip is the sand-blast or using two $6 \mathrm{~mm}$ reinforcement, and the compressive strength of SCC and normal concrete were enhance the shear force.
\end{abstract}




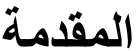

عند صب طبقتين من الخرسانة في المنشأت اما ان تكون خرسانة اعتيادية قديمة مع خرسانة جديدة

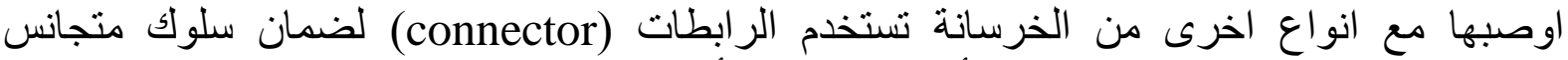

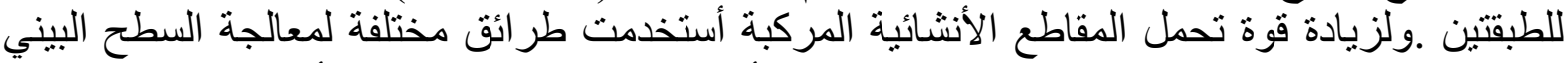

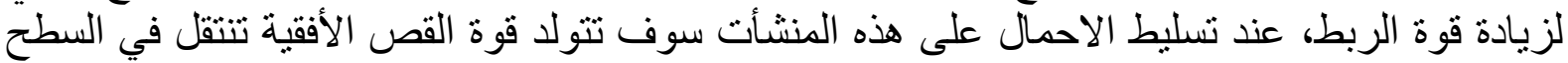

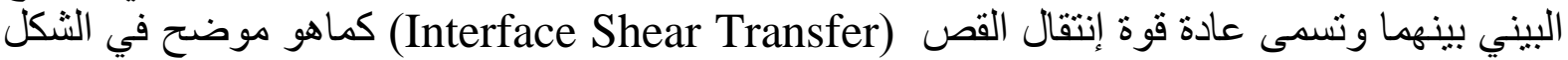

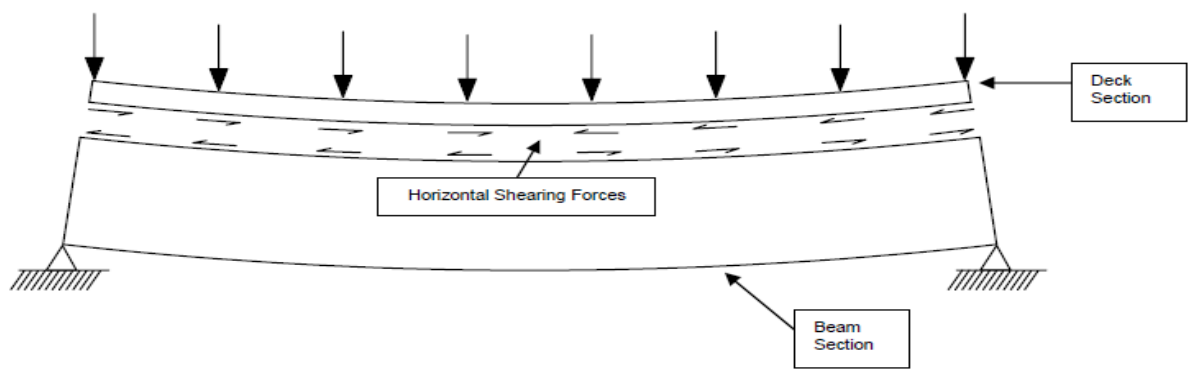

الثكل(1) إنتقال قوة القص بمنطقة الربط بالمقاطع المركبة

تعرف قوةانتقال القص بأنها قوة القص عبر المنطقة الر ابطة (سطح التنلامس) بين الخرسانة ذات الأعمار

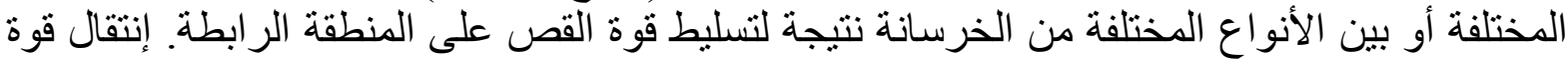

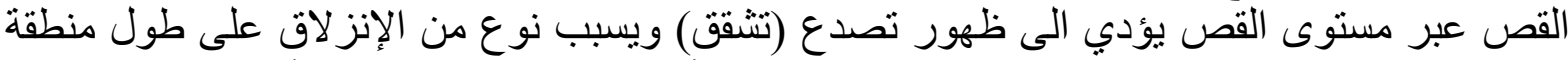

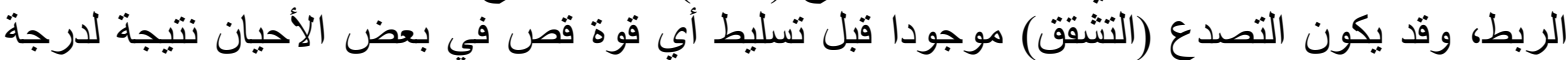

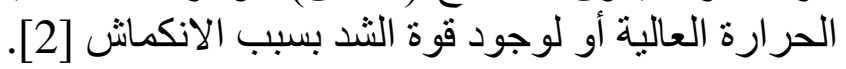

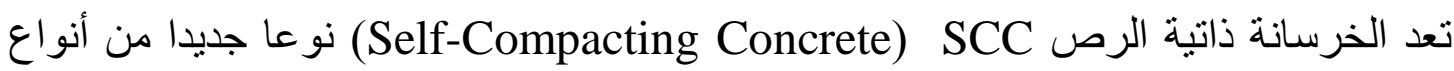

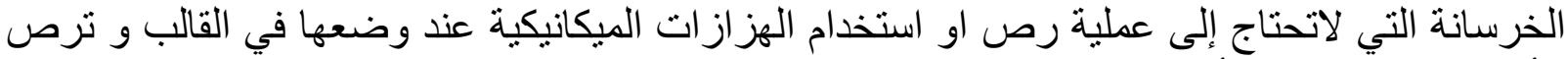

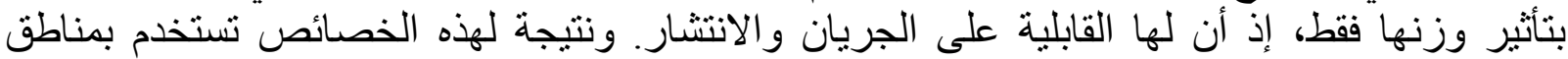

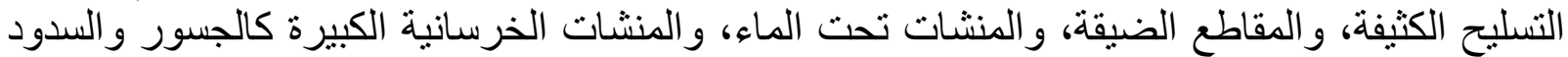

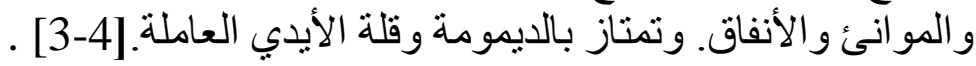

إن الهدف من البحث هو در اسة إنتقال قوة القص بين الخرسانة الأعتبادية المسبقة الصب و الخرسانة ذاتية

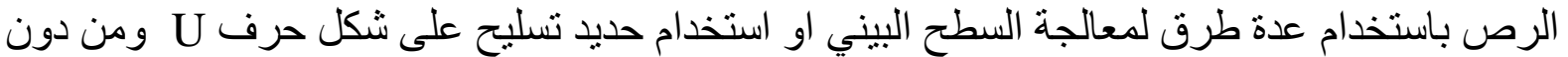

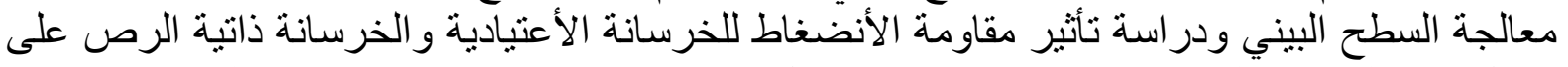

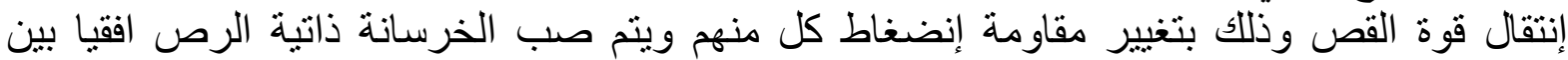

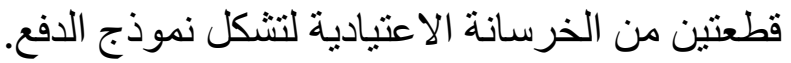

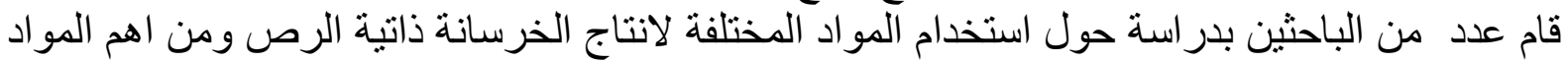

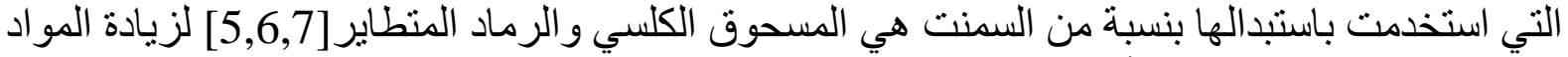

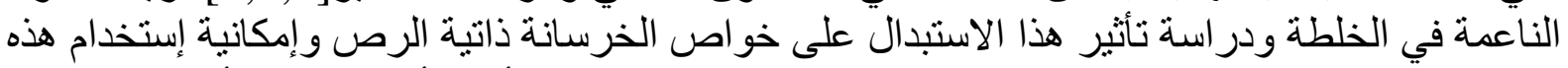

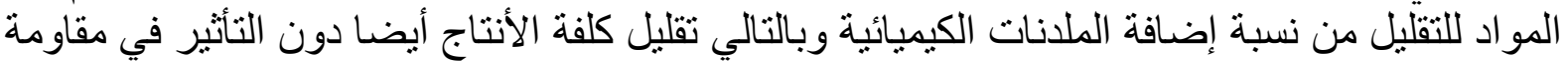

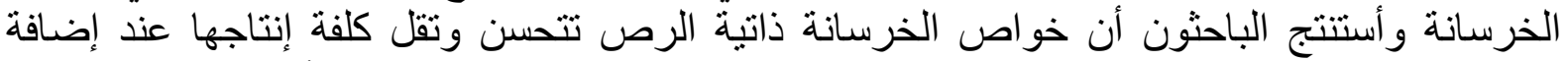

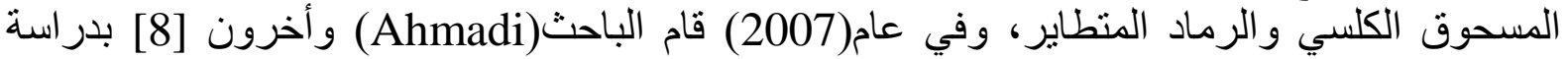

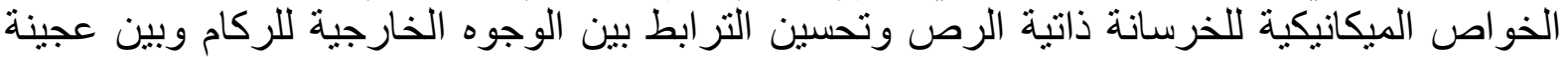

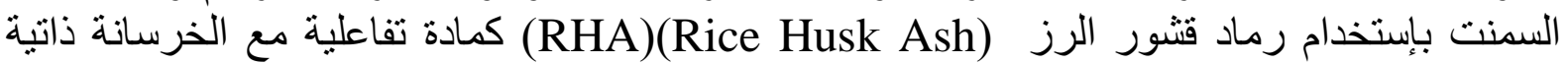


الرص واستتتج ان زيادة رماد قشر الأرز المستبدل بالسمنت يعطي تأثنير كبير ا في الخلطات الخرسانة

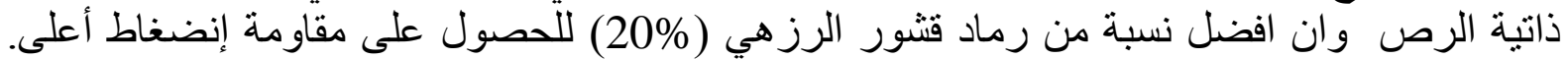

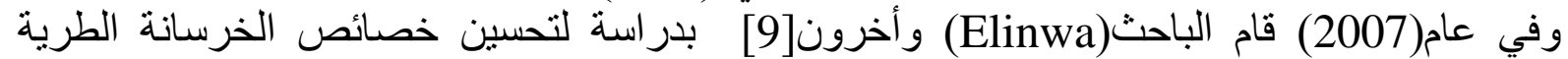
والحصول على خرسانة ذاتية الرص بإستخدام نشارة الخشب كمادة ناعمة (وهي إحدى المخلفات

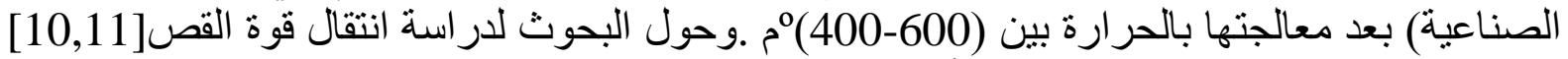

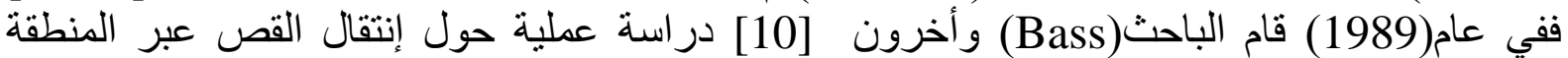
الرابطة بين الخرسانة الاعتيادية القديمة والخرسانة الاعتيادية الجديدة حيث جرى دراسة هذه الحالة

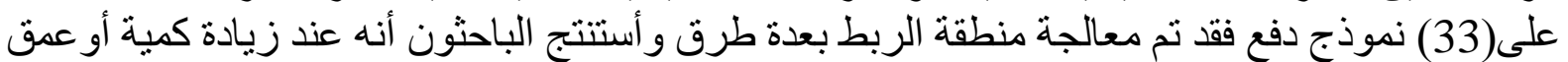
القضبان المدفونة (embedment bars ) داخل الخرسانة ينتج زيادة في قوة إنتقال القص لمنطقة الربط. في عام(2005) قام الباحث (1) [1] بلدراسة أنتقال القص الأفقي بين الخرسانة العالية الأداء (Lightweight concrete) والخرسانة خفيفة الوزن (Ultra High Performance Concrete) بعمل (24) نموذج دفع (push off) ومعالجة السطح للمنطقة الر ابطة بطرق مختلفة وأستتنج الباحث بأن أستخدام الخرسانة العالية الأداء يعطي للجسور قوة وتحمل ومتانة عالية وأستتتج الباحث أن أفضل أنسل طرق المعالجة للسطح البيني عند عدم أستخدام حديد التسليح هي التنقير.

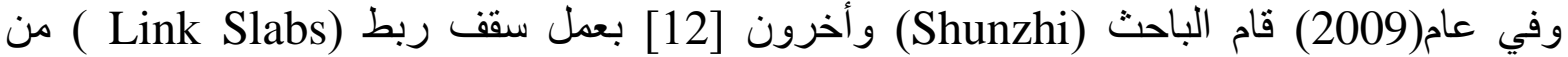

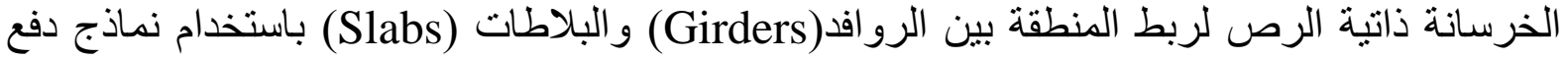
و (Push off)

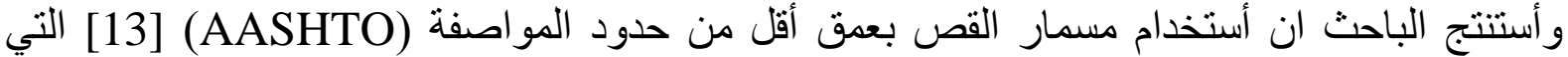

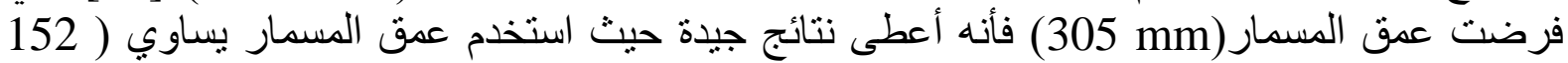

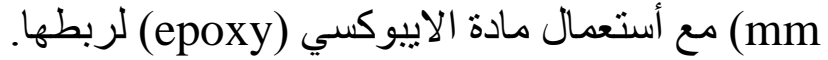

\section{البرنامج العملي والمواد المستخدمة}

تم استخدام اسمنت عراقي محلي الصنع ومطابق للمواصفات العراقية (14] (IQS, NO.5,1984)

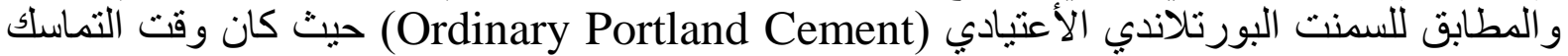

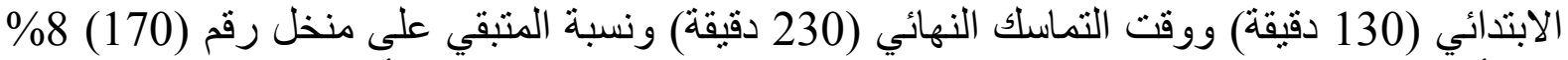

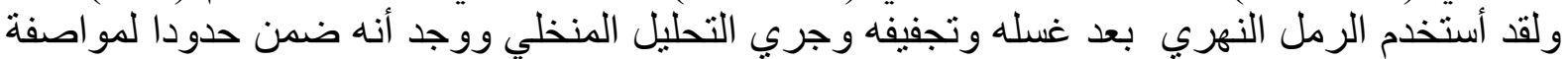

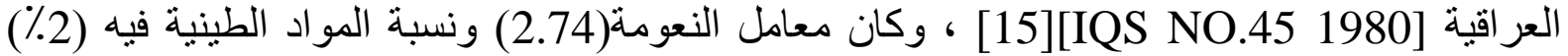
و هي ضدن الحد المسموح به وتم استخدام الحصى النهري كركام خشن ذو مقاس أقصى (19 (19 ملم)،و تم

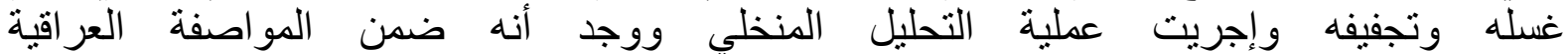

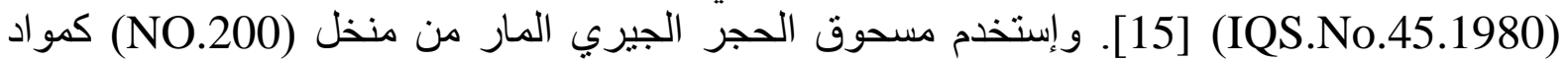
ناعمة (مواد مالئة) كنسبة تعويضية دن السمنت والتي تعمل بدور ها على زيادة قابلية التشغيل للمزحة الخرسانية و أستخدم الملدن الفائق (Sikament -NN) كمضاف لزئه فيادة قابلية تشغيل الخرسانة ذاتية

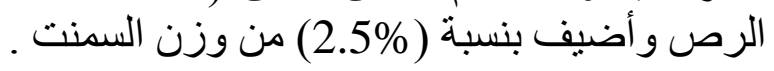

تم إعداد عدد من الخلطات التجريبية بنسب مختلفة وذلك بإستخدام إسطوانات ذات أبعاد ( 300 × 150

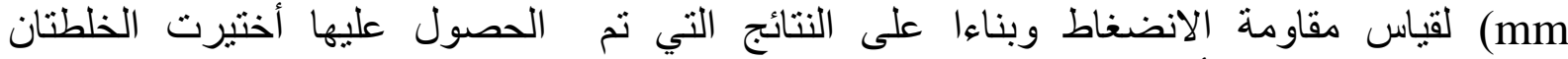

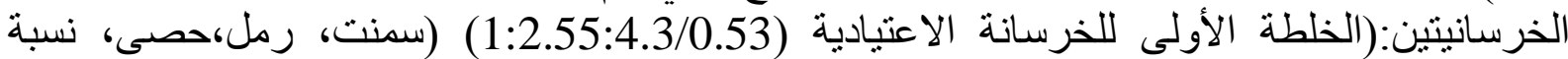

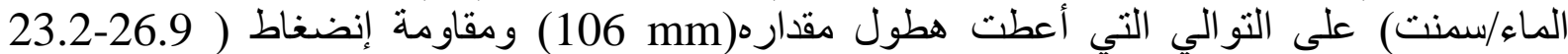
(MPa)، والخلطة الثانية (1:2:2.88/0.45) التي ألتي أعطت هطول مقداره (110mm) ومقاومة إنضغاط للاسطوانة (33.6-38.7 MPa)، و للخرسانة ذاتية الرص فقد تم عمل عدد من الخلطات التجريبية ايضا و أختيرت الخلطتان:(الخلطة الأولى (33.7 (0.92:2.1:1.7:0.36:0.08/2.5) (سمنت، رمل،حصى، نسبة 
ماء/ سمنت، مسحوق الحجر الجيري، نسبة الملدن الفائق من الاسمنت) على التوالي التي أعطت مقاومة

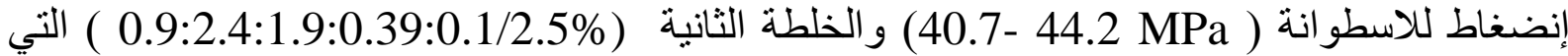
أعطت مقاومة إنضغاط 31.6-37.4 MPa) (16] (16).

خواص الخرسانة ذاتية الرص

لمعرفة الخو اص الطرية للخرسانة ذاتية الرص نم قياس قابلية الانتشار وقابلية الاجتياز وقابلية

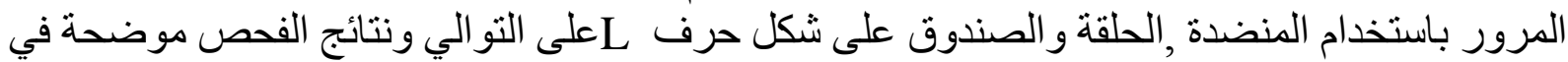

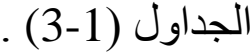

جدول(1) نتائج فحص إنتشار الخرسانة ذاتية الرص

\begin{tabular}{|c|c|c|c|c|c|c|}
\hline Mix & $\begin{array}{c}\mathrm{T}_{500} \\
(\mathrm{sec})\end{array}$ & $\begin{array}{c}\text { Limit } \\
(\mathrm{sec})\end{array}$ & $\begin{array}{c}\mathrm{D}_{\max } \\
(\mathrm{mm})\end{array}$ & $\begin{array}{c}\mathrm{D}_{\text {perp }} \\
(\mathrm{mm})\end{array}$ & $\begin{array}{c}\mathrm{Sj} \\
(\mathrm{mm})\end{array}$ & $\begin{array}{c}\text { Limit } \\
(\mathrm{mm})[17]\end{array}$ \\
\hline 1 & 3 & & 700 & 690 & 690.5 & \multirow{2}{*}{$650-800$} \\
\hline 2 & 2.6 & $2-5$ & 740 & 760 & 750 & $650-800$ \\
\cline { 1 - 3 } & & &
\end{tabular}

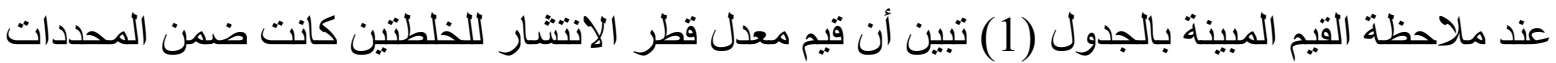
المطلوبة. وكان الزمن المستغرق للوصول إلى القطر (500 mm) للخلطة الأولى أكبر من الزئ من

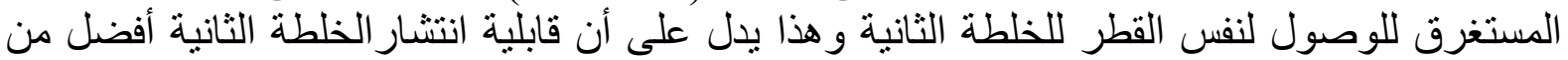
قابلية انتشار الخلطة الأولى ويعود السبب في ذلك إلى أن كمية المو اد الناعمة للخلطة الثانية أكبر من كمية المو اد الناعمة للخلطة الأولى الألى. جدول (2 ) نتائج فحص الحلقة

\begin{tabular}{|c|c|c|c|c|c|c|c|c|c|c|c|}
\hline $\operatorname{mix}$ & $\begin{array}{c}\mathrm{T}_{5} \\
(\mathrm{Sec})\end{array}$ & $\begin{array}{c}\mathrm{D}_{\max } \\
(\mathrm{cm})\end{array}$ & $\begin{array}{c}\mathrm{D}_{\text {perp }} \\
(\mathrm{cm})\end{array}$ & $\begin{array}{c}\mathrm{D}_{\mathrm{av}} \\
(\mathrm{cm})\end{array}$ & $\begin{array}{c}\Delta \mathrm{h}_{\mathrm{x} 1} \\
(\mathrm{~cm})\end{array}$ & $\begin{array}{c}\Delta \mathrm{h}_{\mathrm{x} 2} \\
(\mathrm{~cm})\end{array}$ & $\begin{array}{c}\Delta \mathrm{h}_{\mathrm{y} 1} \\
(\mathrm{~cm})\end{array}$ & $\begin{array}{c}\Delta \mathrm{h}_{\mathrm{y} 2} \\
(\mathrm{~cm})\end{array}$ & $\begin{array}{c}\Delta \mathrm{h}_{0} \\
(\mathrm{~cm})\end{array}$ & $\begin{array}{c}\mathrm{B}_{\mathrm{j}} \\
(\mathrm{mm})\end{array}$ & $\begin{array}{c}\text { Limit } \mathrm{B}_{\mathrm{j}} \\
{[17]}\end{array}$ \\
\hline 1 & 3.2 & 67 & 69 & 68 & 11.5 & 11 & 11.5 & 11.3 & 10.3 & 10.25 & $<15$ \\
\cline { 1 - 7 } & 2.8 & 70 & 72 & 71 & 12.3 & 12.3 & 12.3 & 12.3 & 12 & 8.25 & $\mathrm{~mm}$ \\
\hline
\end{tabular}

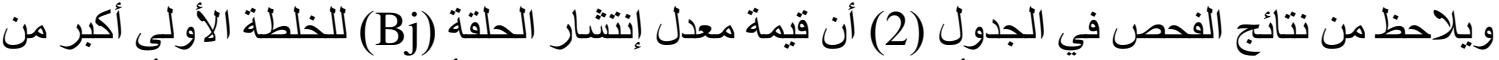

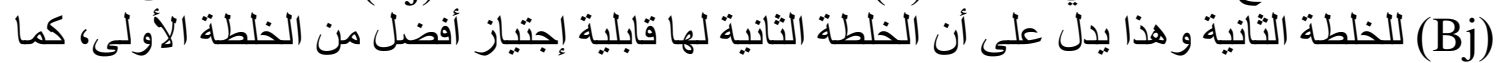

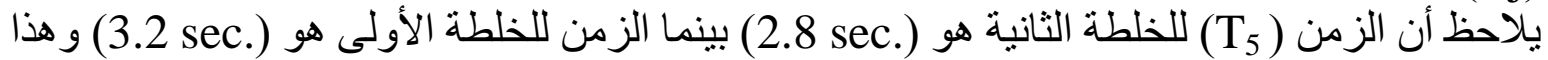
يدل على أن الخلطة الثانية لها قابلية اجتياز أفضل من الخلطة (2. الخطة الأولى.

جدول(3) نتائج فحص الصندوق على شكل حرف(L)

\begin{tabular}{|c|c|c|c|c|}
\hline MIX & $\mathrm{H}_{1}(\mathrm{~cm})$ & $\mathrm{H}_{2}(\mathrm{~cm})$ & $\mathrm{H}_{2} / \mathrm{H}_{1}$ & Limit $\left(\mathrm{H}_{2} / \mathrm{H}_{1}\right)[17]$ \\
\hline 1 & 11 & 8.9 & 0.81 & $0.8-0.85$ \\
\cline { 1 - 3 } & 9 & 7.5 & 0.833 & $0.8-0.85$ \\
\hline
\end{tabular}

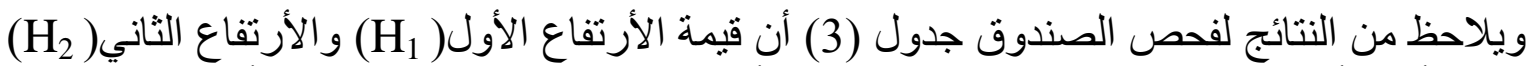

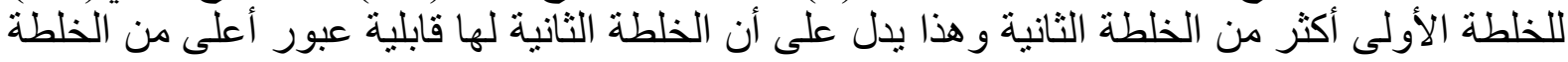

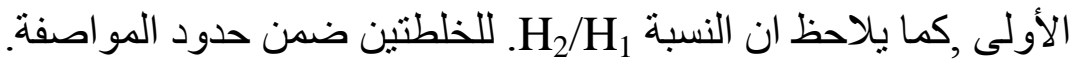


نماذج الافع (Push-Off)

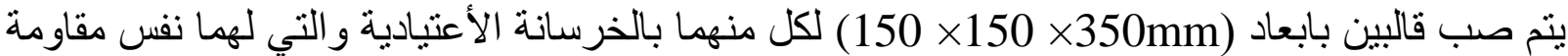

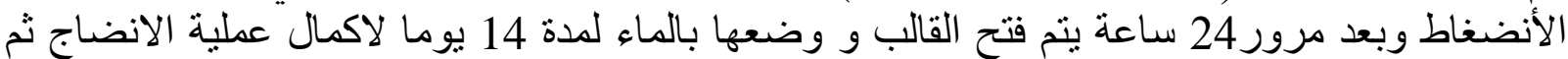
تترك لمدة 60 يوما او اكثر في المختبر، جدول (1)، ونم معالجة السطح البيني بالطرق التالية:

1. مفتاح القص (shear key):تم عمل فراغات منشورية الثكل عدد اثنان في السطح البيني بالا بعاد و الموقع المبين في شكل(2) ويتم عمل مفتاح القص اثناء الصب .
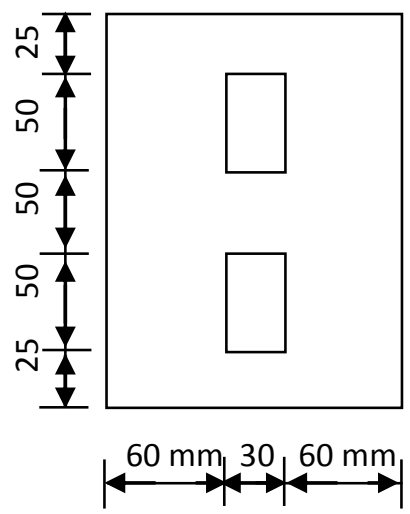

(a) (a) موقع مفتاح القص في السطح البيني

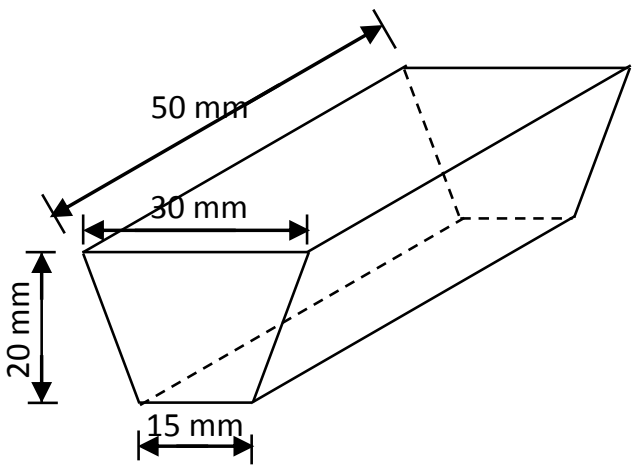

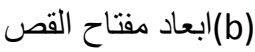

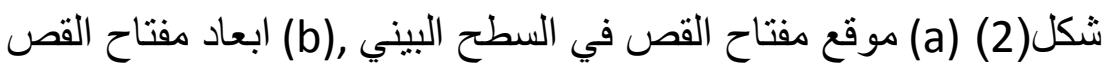

2. الضرب بالرمل(sand blast): تم ضرب مساحة السطح البيني للخرسانة الاعتيادية بعد تصلبها

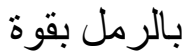
3. التنقير(chipping): تم عمل حفر في السطح البيني بعدق لا يقل عن (3mm) في الخرسـانة الاعتبادية بعد تصلبها. 4. حديد تسليح : تم استخدام حلقتين حديد بقطر 6mm او حلقة واحدة بقطر (6,10mm) على شكل

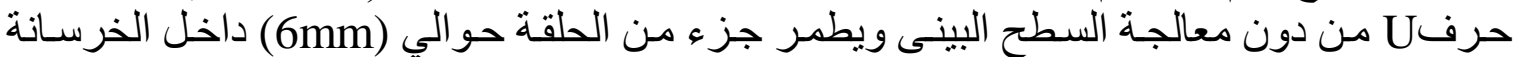
الاعتيادية وتوضع في منتصف السطح البيني كما هو موضح في الثكل(3) .

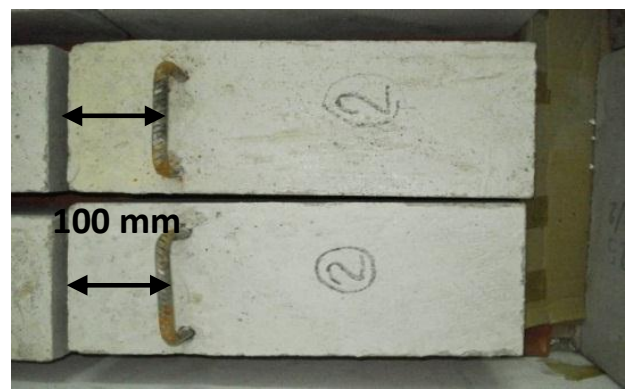

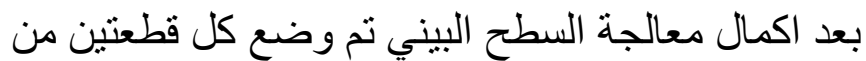

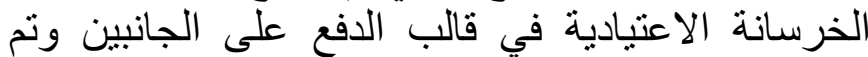

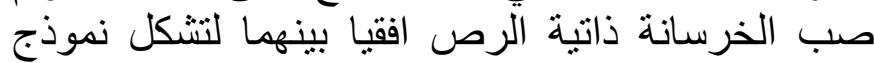

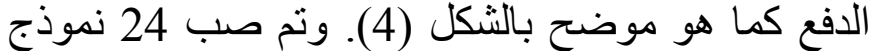

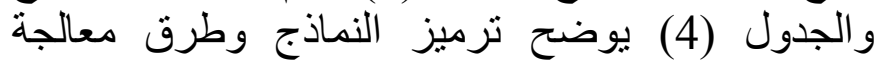
السطح البيني للنماذج.

شكل(3) حلقات التسليح في منطقة القص 


\begin{tabular}{|c|c|c|c|}
\hline Specimens No. & $\begin{array}{c}\text { Age of NC } \\
\text { (days) }\end{array}$ & $\begin{array}{c}\text { Surface } \\
\text { preparation }\end{array}$ & $\begin{array}{c}\text { Shear } \\
\text { reinforcement }\end{array}$ \\
\hline N.SCC.S1 & 71 & $\mathrm{~S}$ & \\
\hline N.SCC.Ch1 & 77 & $\mathrm{Ch}$ & \\
\hline N.SCC.SK1 & 65 & SK & \\
\hline N.SCC.1.R1 & 64 & Smooth & $1 \# 10$ \\
\hline N.SCC.1.R1 & 59 & Smooth & $1 \# 6$ \\
\hline N.SCC.2.R1 & 59 & Smooth & $2 \# 6$ \\
\hline N.SCC.S2 & 64 & $\mathrm{~S}$ & \\
\hline N.SCC.Ch2 & 79 & $\mathrm{Ch}$ & \\
\hline N.SCC.SK2 & 73 & SK & \\
\hline N.SCC.1.R2 & 60 & Smooth & $1 \# 10$ \\
\hline N.SCC.1.R2 & 57 & Smooth & $1 \# 6$ \\
\hline N.SCC.2.R2 & 73 & Smooth & $2 \# 6$ \\
\hline N.SCC.S3 & 73 & $\mathrm{~S}$ & \\
\hline N.SCC.Ch3 & 78 & $\mathrm{Ch}$ & \\
\hline N.SCC.SK3 & 64 & SK & \\
\hline N.SCC.1.R3 & 62 & Smooth & $1 \# 10$ \\
\hline N.SCC.1.R3 & 58 & Smooth & $1 \# 6$ \\
\hline N.SCC.2.R3 & 60 & Smooth & $2 \# 6$ \\
\hline N.SCC.S4 & 66 & $\mathrm{~S}$ & \\
\hline N.SCC.Ch4 & 80 & $\mathrm{Ch}$ & \\
\hline N.SCC.SK4 & 73 & SK & \\
\hline N.SCC.1.R4 & 58 & Smooth & $1 \# 10$ \\
\hline N.SCC.1.R4 & 58 & Smooth & $1 \# 6$ \\
\hline N.SCC.2.R4 & 64 & Smooth & $2 \# 6$ \\
\hline
\end{tabular}

Note: S: Sand blasted, Ch: Chipped , SK: Shear Key, 1\#10: one stirrup of 10mm diameter, 1\#6: one stirrup of $6 \mathrm{~mm}$ diameter.N: normal conc.R:reinforcement

وتم تسليط الاحمال على القطعة الوسطية والتي تمثل الخرسانة ذاتية الرص باستخدام جهاز هيدروليكي ذو التون

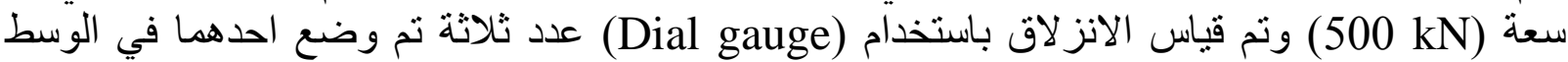
و اثنان على الجوانب كما هو موضح في الثكل (5) (5). 


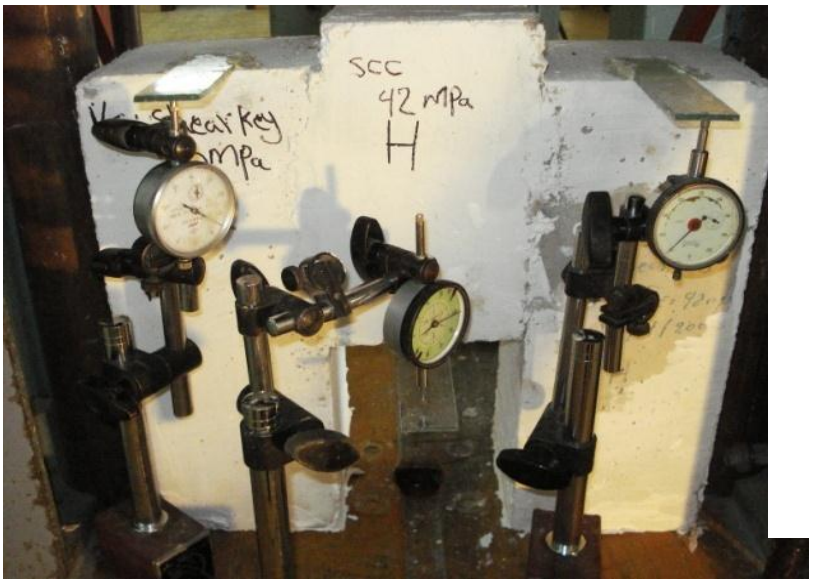

شكل(5) طريقة قياس الانزلاق

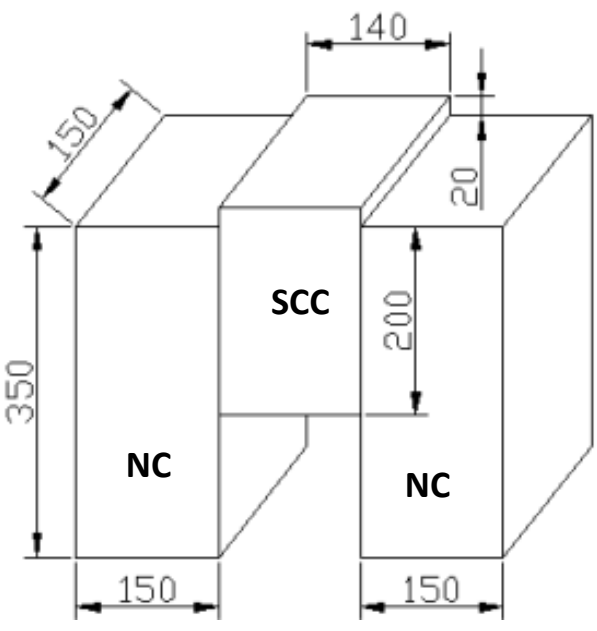

(all dimension in $\mathrm{mm}$ ) شكل(4) ابعاد نموذج الدفع

\section{نتائج الفحص ومناقشتها}

\section{1 - 1 -نتائج فحص النماذج غير المسلحة}

تم فحص النماذج المعالجة بعمل مفتاح القص, متكونة من(4) نماذج تكون مقاومة الأنضخاط مختلفة كما

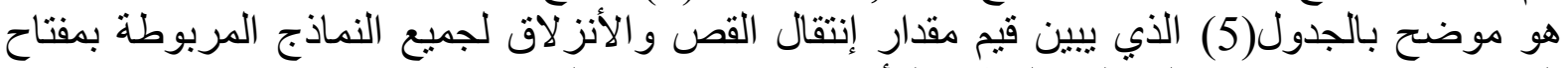

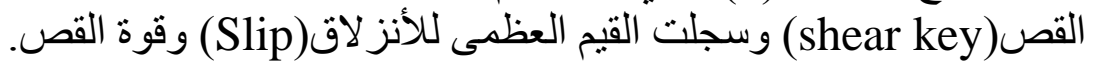

ويوضح الجدول ان اعلى قيمة لقوة القص عند الربط بين الخرسانة الأعتيادية ذات مقاومة الأنضغاط

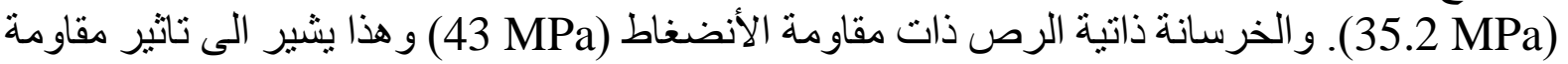
الانضغاط لكل منهما على قوة القصان.

جدول(5) نتائج فحص النماذج المعالجة بمفتاح القص

\begin{tabular}{|c|c|c|c|c|}
\hline $\begin{array}{c}\text { الأنز لاق (Slip) } \\
\text { (mm) }\end{array}$ & $\begin{array}{c}\text { قوة إنتقال القص } \\
\text { (Shear force) } \\
(\mathrm{kN})\end{array}$ & للخرس الرسانة ذاتية & $\begin{array}{c}\text { للخرسانة الأعنيادية } \\
\text { (MPa) }\end{array}$ & نوع المعالجة \\
\hline 0.0125 & 20 & 36.6 & 26.1 & \multirow{4}{*}{$\begin{array}{c}\text { مفتاح القص (Shear key) } \\
\text { (Shear }\end{array}$} \\
\hline 0.018 & 22.5 & 41.3 & 26.1 & \\
\hline 0.0135 & 25.5 & 31.6 & 35.2 & \\
\hline 0.0179 & 28.5 & 43 & 35.2 & \\
\hline
\end{tabular}

كما تم فحص النماذج المعالجة بالتنقير متكونة من(4) نماذج ذوات مقاومة الأنضغاط مختلفة كما هو

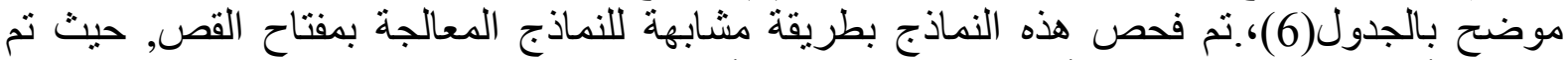
تسليط الأحمال تدريجيا وقياس الأنزلاق عند كل قيمة للأحمال. 


\begin{tabular}{|c|c|c|c|c|}
\hline $\begin{array}{c}\text { (Slip) الأنز لاق) } \\
\text { (mm) }\end{array}$ & $\begin{array}{c}\text { قوة إنتقال القصال } \\
\text { (Shear force) } \\
(\mathrm{kN})\end{array}$ & مقاومة الأنضغاط الرانة ذاتية & $\begin{array}{c}\text { للخرسانة الأعنيادية } \\
\text { (MPa) }\end{array}$ & نوع المعالجة \\
\hline 0.0218 & 37.5 & 33.5 & 26.9 & \multirow{4}{*}{$\begin{array}{c}\text { التنقير } \\
\text { (Chipped) }\end{array}$} \\
\hline 0.031 & 42.5 & 41.2 & 26.9 & \\
\hline 0.0372 & 50 & 33.5 & 36.6 & \\
\hline 0.0428 & 60 & 41.6 & 36.6 & \\
\hline
\end{tabular}

كما تم فحص النماذج المعالجة بالضرب بالرمل ومتكونة من(4) نماذج ومقاومة انضخاط مختلفة كما هو

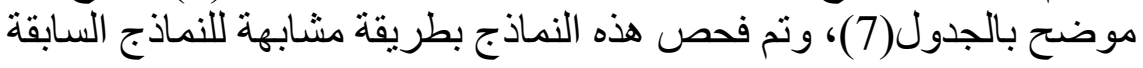

\begin{tabular}{|c|c|c|c|c|}
\hline $\begin{array}{l}\text { (Slip) الأنز لاق) } \\
\text { (mm) }\end{array}$ & $\begin{array}{c}\text { قوة إنتقال القص } \\
\text { (Shear force) } \\
(\mathrm{kN})\end{array}$ & اللخرسانة ذانية الأنضغاط & $\begin{array}{c}\text { للخرسانة الأنغتيادية } \\
\text { (MPa) }\end{array}$ & نوع المعالجة \\
\hline 0.0367 & 92.5 & 37.4 & 23.2 & \multirow{4}{*}{$\begin{array}{l}\text { الضرب بالرمل } \\
\text { (sand blast) }\end{array}$} \\
\hline 0.039 & 95 & 41.4 & 23.2 & \\
\hline 0.04 & 99 & 33.6 & 37.5 & \\
\hline 0.0431 & 112.5 & 43 & 37.5 & \\
\hline
\end{tabular}

يلاحظ من الجدول أن أعلى قيمة لقوة القص هي عند مقاومةالانضغاط للخرسانة الأعتيادية (37.5MPa) و الخرسانة ذاتية الرص ذات مقاومة إنضغاط (43 MPa) و هذا يدل على ان لمقاومة الانضغاط تاثير

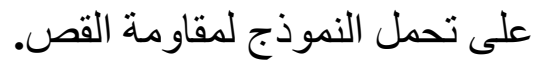

وللمقارنة بين الطرق الثلاثة للمعالجة(مفتاح القص,التنقير و الضرب بالرمل) رسمت العلاقة بين قوة

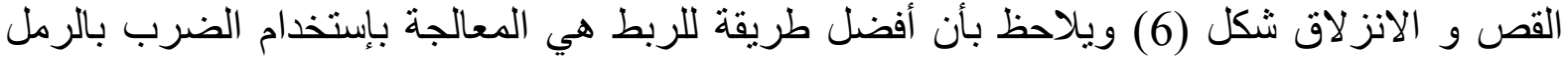

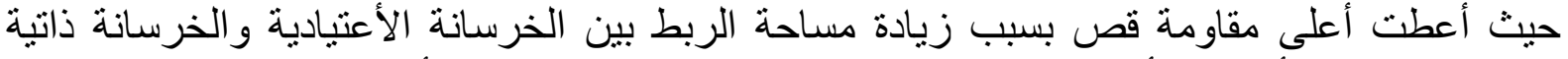

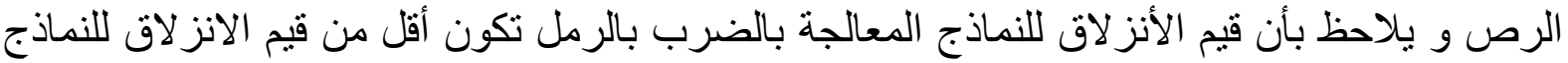

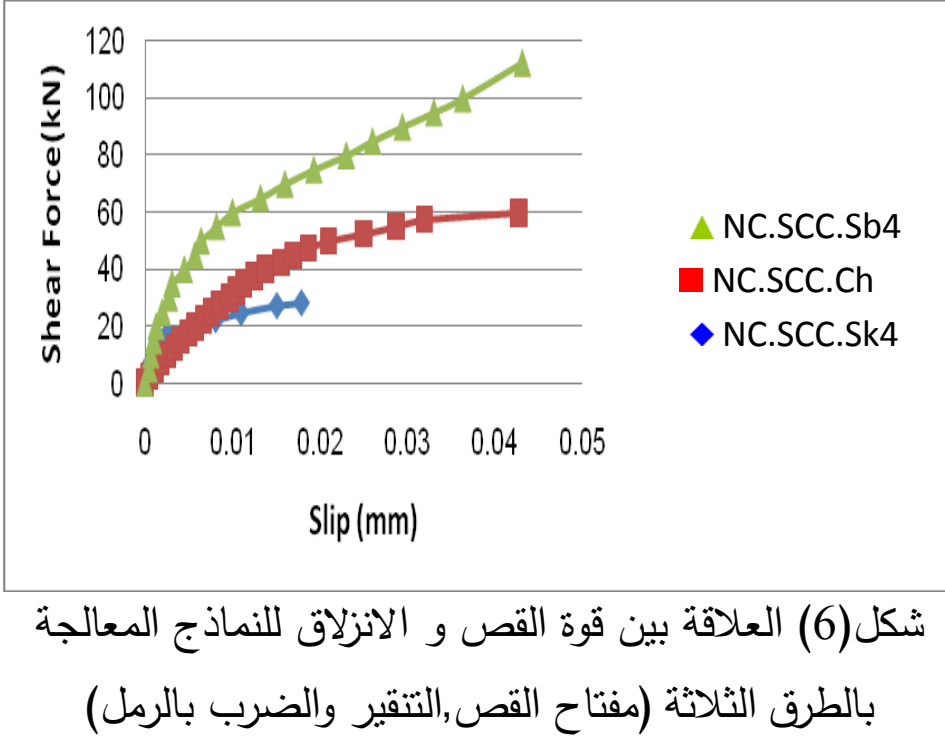

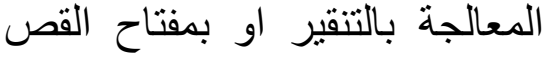

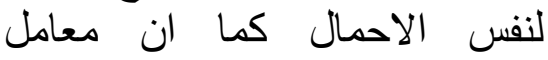
المرونة للقص والتي تمثل ميلان الفيان

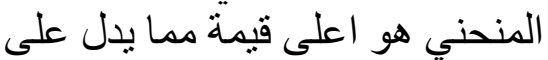

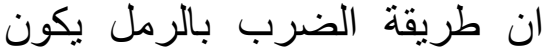
النموذج اكثر صلادة .

تحتوي على حديد تسليح النماذج التي تم فحص (4) نماذج تحتوي في لاكي منطقة الربط الاملس تسليح قص تصن في

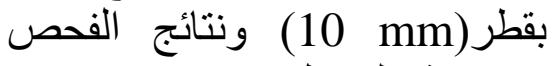
موضحة بالجدول(8). 
كما تم فحص (4) نماذج تحتوي على حلقتين حديد تسليح بقطر (6mm) ومقاومة انضغاط مختلفة

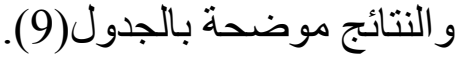

\begin{tabular}{|c|c|c|c|c|}
\hline $\begin{array}{c}\text { الأنز لاق (mm) } \\
\text { (mm) }\end{array}$ & $\begin{array}{c}\text { قوة إنتقال القصال } \\
\text { (Shear force) } \\
(\mathrm{kN})\end{array}$ & اللخرسانة ذاتية الأتضغاط & $\begin{array}{c}\text { للخرسانة الأعتبادية } \\
\text { (MPa) }\end{array}$ & نوع المعالجة \\
\hline 0.04315 & 55 & 33 & 24.3 & \multirow{4}{*}{ قطر الربط بحديد } \\
\hline 0.0471 & 62.5 & 40.7 & 24.3 & \\
\hline 0.05315 & 64 & 33 & 38.7 & \\
\hline 0.055 & 68 & 40.7 & 38.7 & \\
\hline
\end{tabular}

جدول(9) نتائج فحص النماذج التي تحتوي على حلقتين من حديد التسليح بقطر (6mm)

\begin{tabular}{|c|c|c|c|c|}
\hline $\begin{array}{c}\text { الأنز لاق (mm) } \\
\text { (mm) }\end{array}$ & $\begin{array}{c}\text { قوة إنتقال القص } \\
\text { (Shear force) } \\
\text { (kN) }\end{array}$ & 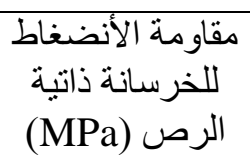 & 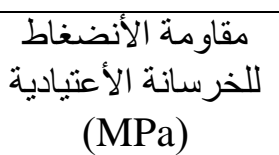 & نوع المعالجة \\
\hline 0.0258 & 52.5 & 33.6 & 26.7 & \multirow{4}{*}{ قديط التسليح بحلتين } \\
\hline 0.0288 & 45 & 41.4 & 26.7 & \\
\hline 0.0311 & 65 & 31.6 & 35.6 & \\
\hline 0.047 & 73.5 & 43.7 & 35.6 & \\
\hline
\end{tabular}

ويلاحظ من الجدولين ان قوة القص للنماذج التى تحتوي على حلقتين بقطر 6mm اعلى من قوة القص وادص للنماذج المسلحة بحلقة واحدة بقطر 10 mm والانزلاق اقل و السبب هو زيادة نسبة التسليح في منطقة

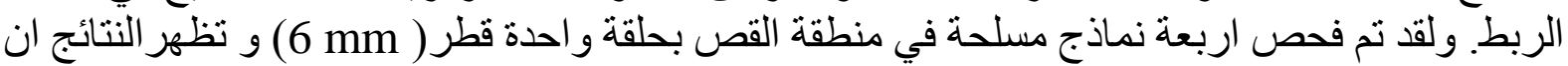
قوة القص لهذه المنماذج اقل من قوة القص للنماذج ذات التسليح بحلقة واحدة قطر (6mm (10 m) او او التسليح بحلقتين قطر (6mm), جدول (10)

جدول(10) نتائج فحص النماذج المسلحة بحلقة قطر (6 mm)

\begin{tabular}{|c|c|c|c|c|}
\hline $\begin{array}{c}\text { (Slip) الأنز لاق) } \\
\text { (mm) }\end{array}$ & $\begin{array}{c}\text { قوة إنتقال القص } \\
\text { (Shear force) } \\
(\mathrm{kN})\end{array}$ & اللخرسانة ذاتي الأنضغاط & $\begin{array}{c}\text { للخرسانة الأعتيادية } \\
\text { (MPa) }\end{array}$ & نوع المعالجة \\
\hline 0.0266 & 28.25 & 37.4 & 26 & \multirow{4}{*}{$\begin{array}{c}\text { الربط بحلقة حديح قطر } \\
\text { التسليد } \\
\text { (6 mm) }\end{array}$} \\
\hline 0.0294 & 30 & 44.2 & 26 & \\
\hline 0.031 & 33.5 & 33.1 & 34.9 & \\
\hline 0.0372 & 40 & 43.7 & 34.9 & \\
\hline
\end{tabular}

و عند المقارنة بين أعلى قيمة لقوة إنتقال القص بين النماذج المسلحة بمنطقة القص كما هو موضح

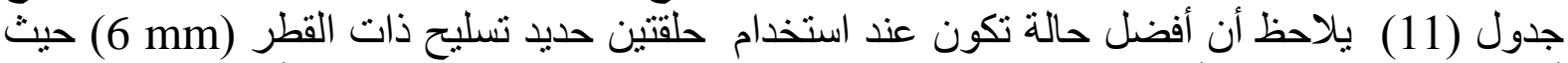
أعطت قوة إنتقال قص أعلى من إستخدام حلقة حديد تسليح ذات قطر (10 mm) و أستخدام حلقة حديد

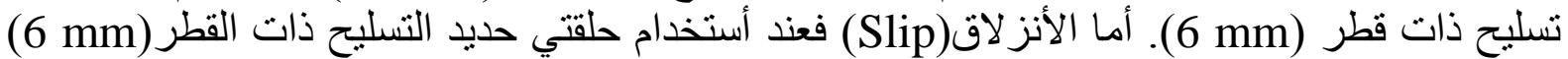

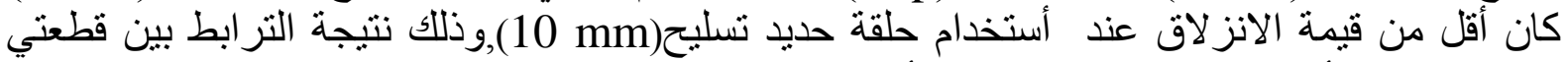

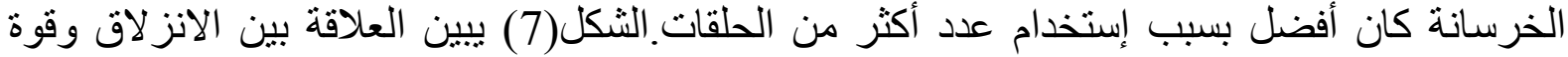
القص للنماذج المسلحة بمنطقة القص , ويلاحظ بأن قيم الأنزلاق عند إستخدام حلقتين حديد تسليح 
قطر(6 mm) يكون أقل من قيم الأنزلاق عند أسخدام حلقة حديد التسليح ذو قطر (mm' mm) وقطر 6 (mm ولنفس قوة القص . جدول(11) قيم قوة القص العظمى و الأنز لاق للنماذج المسلحة بمنطقة القص

\begin{tabular}{|c|c|c|c|c|}
\hline $\begin{array}{c}\text { الأنزلاق (Slip) } \\
\text { (mm) }\end{array}$ & $\begin{array}{c}\text { قوة إنتقال القص } \\
\text { (Shear force) } \\
(\mathrm{kN})\end{array}$ & للخرسانة ذاتية الأنضاط & 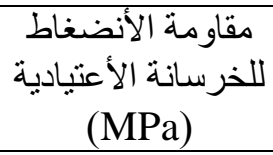 & تسليح القص \\
\hline 0.055 & 68 & 40.7 & 38.7 & حلقة قطر (10 mm) \\
\hline 0.047 & 73.5 & 43.7 & 35.6 & حلقتين قطر (6 mm) \\
\hline 0.0372 & 40 & 43.7 & 34.9 & حلقة قطر (6 mm) \\
\hline
\end{tabular}

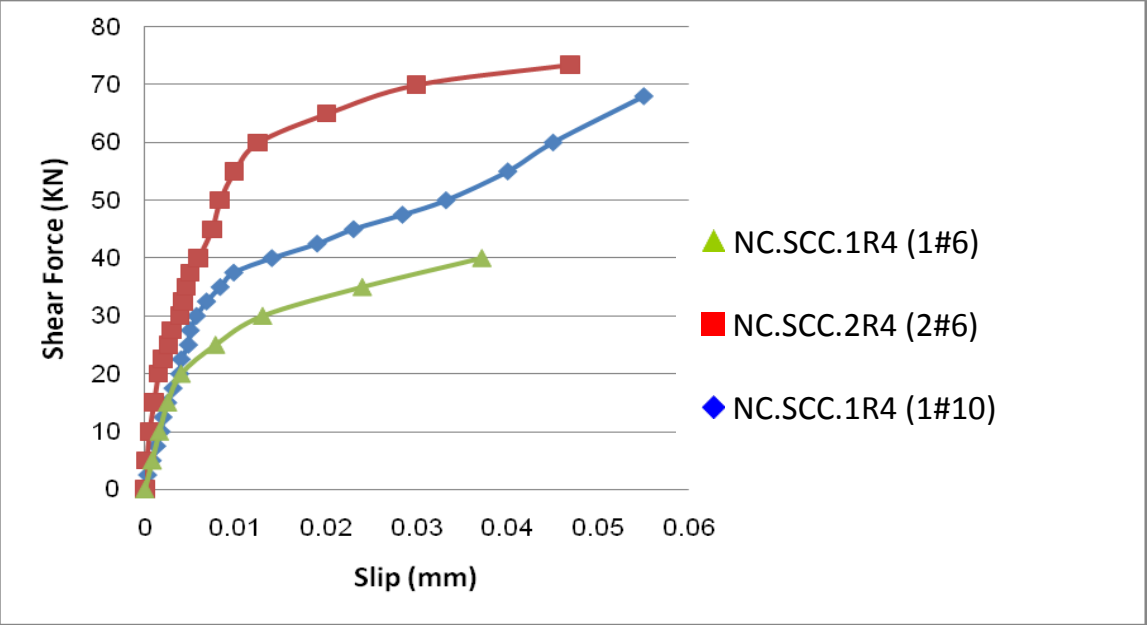

شكل (7) مقارنة قوة القص والانزلاق للنماذج المسلحة بمنطقة القص
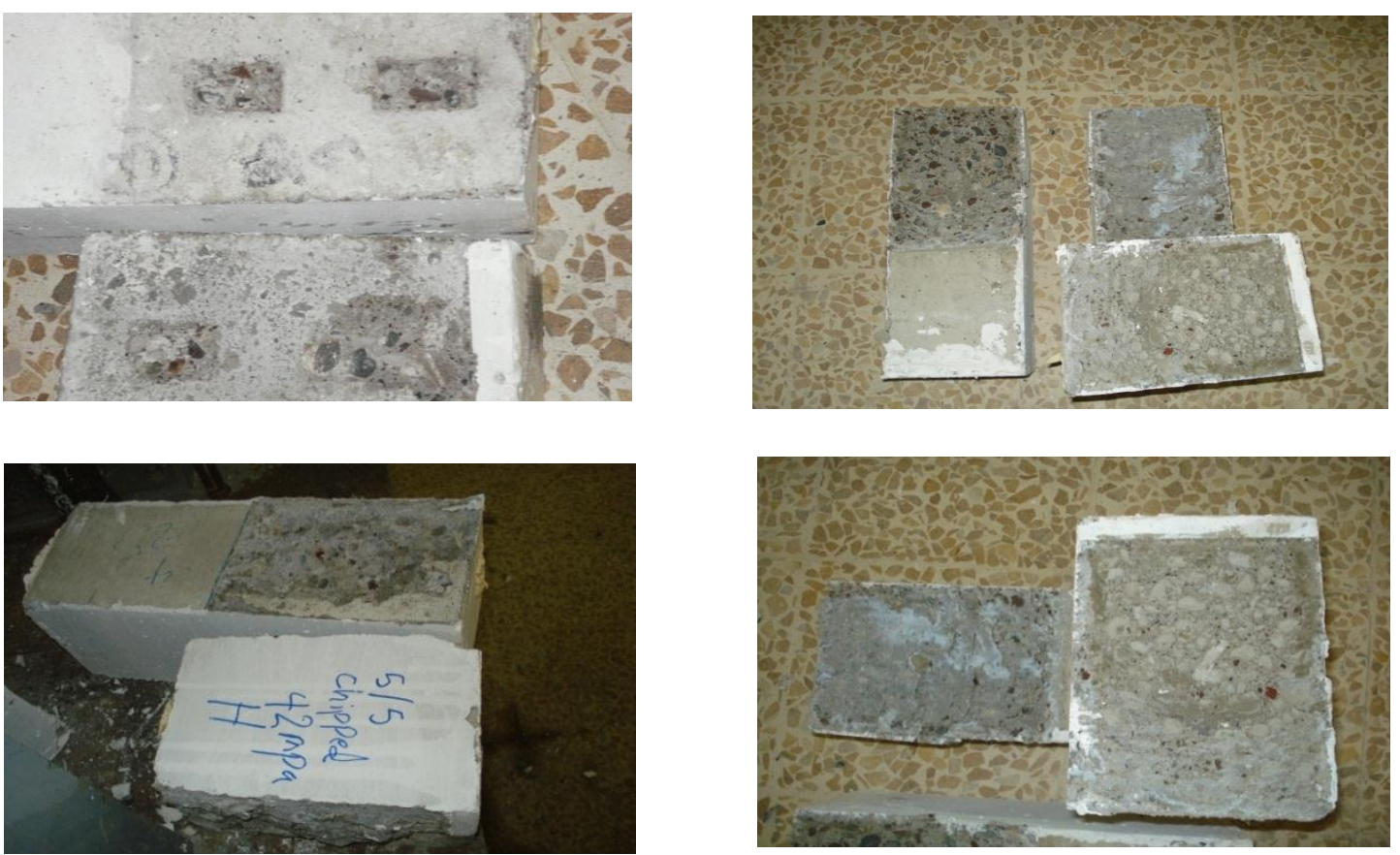

شكل(8) شكل الفشل للنماذج غير المسلحة 


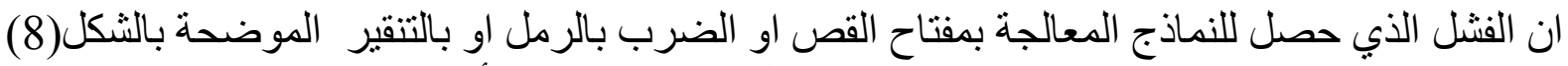

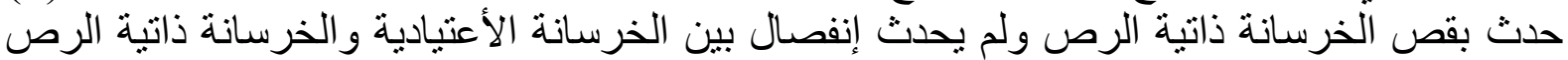

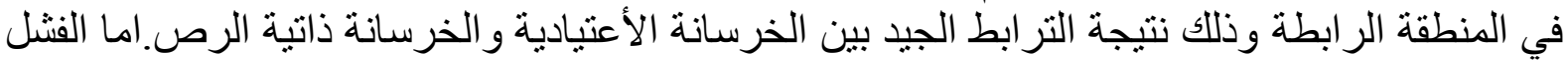

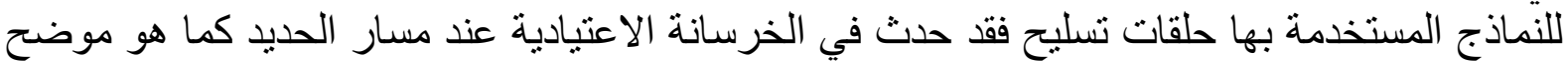

بالثنكل (9) ب (ل)

$$
\text { الاستتناجات }
$$

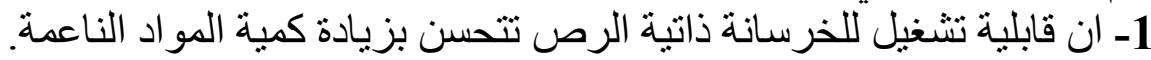

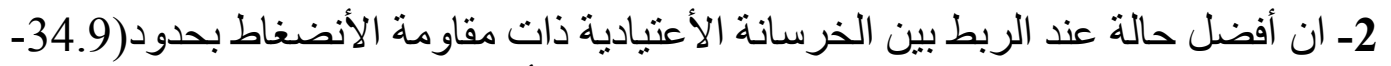

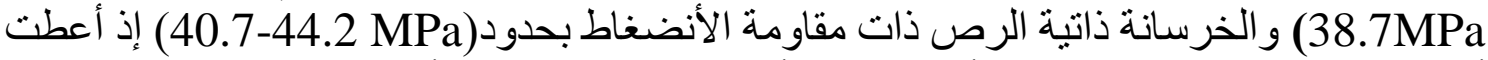
أعلى قوة تحمل للقص و هذا يبين أن مقاومة الأنضغاط للخرسانة لها تأثثر و اضح على قيمة مقاومة

3- ان افضل طريقة لمعالجة السطح البيني للحصول على اعظم قوة قص و اقل انز لاق هي عند الفي

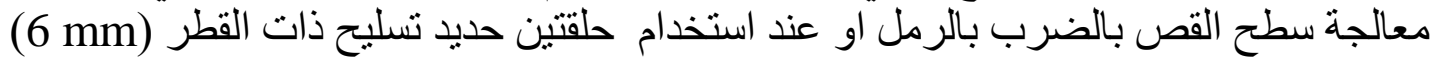

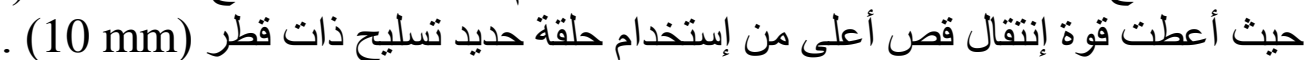

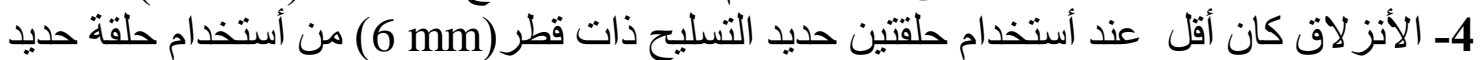

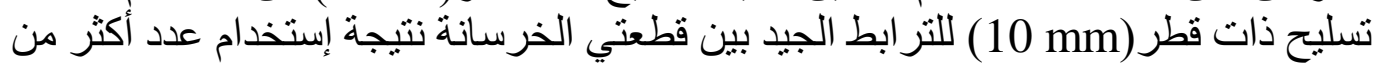

الحلقات في منطقة القص. انص

\section{المصادر}

1. Banta,T.,E., "Horizontal Shear Transfer Between Ultra High Performance Concrete and Lightweight Concrete", M.Sc. Thesis in Civil Engineering, February 2005, Virginia Polytechnic Institute

2. AL-Shammari,M.K. "Shear Strength of Self Compacting Concrete Beams without Web Reinforcement" M.Sc.Thesis, University of Technology,2007.

3. Collepardi, M., Borsoi, A., Collepardi, S., and Troli, R "Strength, shrinkage and creep of SCC and flowing concrete", Second North American Conference on the Design and Use of Self-Consolidating Concrete, Fourth International RILEM Symposium on Self-Compacting Concrete, Edited by Shah, 2005, pp. 911-919

4. Abdul-Hameed, M. " A study of Mix Design and Durability of Self Compacting Concrete" M.Sc.Thesis, King Fahd University of Petroleum and Minerals, Saudi Arabia,2005.

5. Bager, D. H., Geiker, M. R., and Jensen, R. M., "Rheology of SelfCompacting Mortars-Influence of Particle Grading”, NCF,29-4, 2008, pp. 1 $-14$

6. Felekoglu, B., Turkel, S., and Baradan, B., Effect of water/cement ratio on the Fresh and Hardened Properties of Self-Compacting Concrete, Building and Environment, Vol. 42, 2007 , pp. 1795-1802 
7. ALharbi,M, Effect of Different Curing Conditions on Some Properties of Self- Compacting Concrete, M.Sc. Thesis, Mosul University, Iraq,2008.

8. Ahmadi, M., Alidoust, O., Sadrinejad, I., and Nayeri, M., "Development of Mechanical Properties of Self-Compacting Concrete Contain Rice Husk Ash". Proceedings of World ACADEMY of SCIENCE Engineering and Technology, Vol.23, ISSN 1307-6884, August, 2007, pp.503-506.

9. Elinwa, A. U., Ejeh, S. P., And Mamuda, A. M., "Assessing Of th Fresh Concrete Properties Of Self-Compacting Concrete Containing Sawdust Ash", Construction And Building Materials, 6 February, 2007 , pp.1-5

10. Bass,R.A. Ramon L. Carrasquillo, and James O. J. "Shear Transfer Across New and Existing Concrete Interfaces" ACI Structural Journal No. 86-S34 July-August 1989

11. Choi,R.A. "Shear Transfer Across New and Existing Concrete Using Large Powder-Driven Nail " ACI Structural Journal, Vol.96,No.2, March-April, 1999, pp. 183-192.

12. Shunzhi Q., Michael D. Lepech, K., And Victor C. Li " Introduction Of Transition Zone Design For Bridge Deck Link Slabs Using Ductile Concrete" ACI Structural Journal No. 106-S11 January-February 2009.

13. AASHTO LRFD " Bridge Design Specification", American Associate of State Highway and Transportation Official,Washington,D.C., 2004.

14. الجهاز المركزي للتقييس و السيطرة النوعية،"المو اصفات القياسية (رقم 5) "خصائص الأسمنت

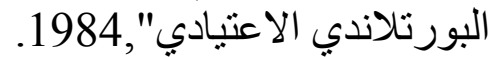

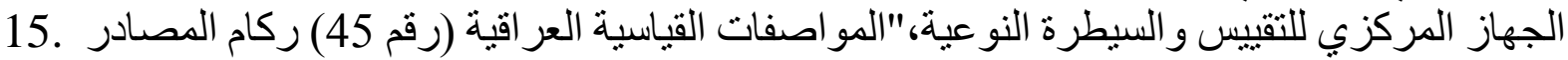

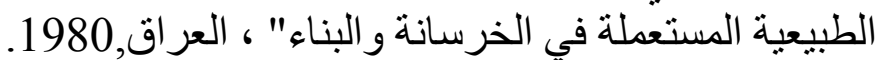

16. Al-hadide, R.S., "Shear Transfer at the Interface between Precast Normal Concrete and Self-Compacting Concrete", M.Sc.Thesis, Mosul University, Iraq, 2009.

17. ASTM C1611 "Standard Test Method for Self-Compacting Concrete" Philadelphia, Pennsylvania.

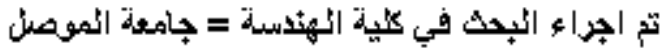

